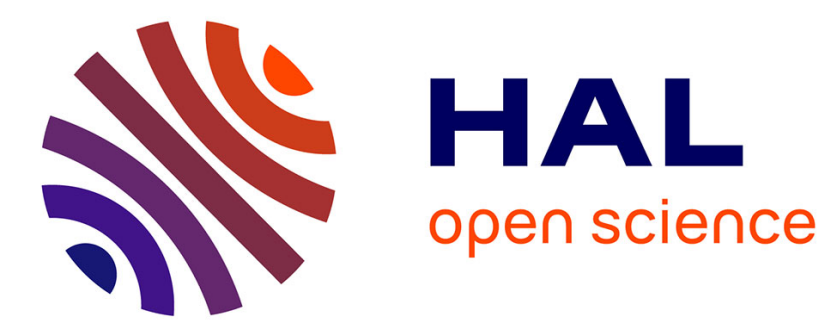

\title{
Observer design for nonlinear systems under unknown time-varying delays
}

\author{
Malek Ghanes, Jesus de Leon, Jean-Pierre Barbot
}

\section{To cite this version:}

Malek Ghanes, Jesus de Leon, Jean-Pierre Barbot. Observer design for nonlinear systems under unknown time-varying delays. IEEE Transactions on Automatic Control, 2013, 58 (6), pp.1529 1534. 10.1109/TAC.2012.2225554 . hal-00749682

\section{HAL Id: hal-00749682 \\ https://hal.inria.fr/hal-00749682}

Submitted on 8 Nov 2012

HAL is a multi-disciplinary open access archive for the deposit and dissemination of scientific research documents, whether they are published or not. The documents may come from teaching and research institutions in France or abroad, or from public or private research centers.
L'archive ouverte pluridisciplinaire HAL, est destinée au dépôt et à la diffusion de documents scientifiques de niveau recherche, publiés ou non, émanant des établissements d'enseignement et de recherche français ou étrangers, des laboratoires publics ou privés. 


\title{
Observer design for nonlinear systems under
}

\section{unknown time-varying delays}

\author{
M. Ghanes, J. De Leon and J-P. Barbot
}

\begin{abstract}
The design of observers for nonlinear systems with unknown, time-varying, bounded delays, on both state and input, still constitutes an open problem. In this paper, we show how to solve it for a class of nonlinear systems by combining the high gain observer approach with a Lyapunov-Krasovskii functional suitable choice. Sufficient conditions are provided to prove the practical stability of the observer. It is shown that the observation error is bounded and depends on the size of two parameters: the known upper bound delay of the unknown time-varying function delay and the instantaneous state dynamic variation. Furthermore, for the particular case of constant known time delay, the convergence of the proposed observer becomes exponential. The feasibility of the proposed strategy is illustrated by a numerical example.
\end{abstract}

\section{INTRODUCTION}

It is well-known that time delay is an inherent property of several dynamical systems such as in tele-operation, communications, mechanical, electrical, biological embedded systems and many others ( [2], [6], [24], [26], [27], [31], [33], [37]). However, to reconstruct the state of such systems, it has only been solved for particular cases and a large variety of open problems concerning nonlinear observer for time delay system exist. For this reason, the observer design for time-delay nonlinear systems has been attracting the attention for researchers in recent years. Furthermore, important efforts have been done to solve such problem and different observation approaches have been employed. For example, those based on asymptotic approach ( [4], [9], [18], [19]), exponential method ( [5], [8], [10], [20], [28]), numerical approach ( [3], [26]), algebraic method ( [1], [11], [12], [25], [40], [41]), sliding mode approach

M. Ghanes and J.P. Barbot are with ECS-Lab/ENSEA, France. e-mail: ghanes@ensea.fr, barbot@ensea.fr

J. De Leon is with the FIME-UANL, Universidad Autonoma de Nuevo Leon; Mexico. e-mail: drjleon@gmail.com

J.P. Barbot is also with EPI Non-A, INRIA, Lille Nord Europe. 
( [13], [32], [35]), $H_{\infty}$ method ( [7], [34], [39]) and so on, which have been developed for linear ( [4], [5], [7], [8], [9], [11], [19], [28], [34], [35], [39]) and nonlinear ( [1], [10], [18], [20], [25], [40], [41]) time-delay systems.

To solve the observation problem, this paper deals with the convergence analysis of an observer for a class of time-delay nonlinear system in strict triangular form, by using an asymptotic observation method.

On the other hand, regarding the time-delay assumptions, in [1] the identifiability of the delay parameter is related to the form of input-output equations for nonlinear systems assuming a single constant known time delay. Furthermore, a high gain observer for unknown time delay nonlinear system in triangular form which an exponential convergence of the observation error has been proposed in [10]. However, the time delay is still considered same for original system and its observer. In [18] a state observer for single-input single-output nonlinear delay systems has been proposed. Conditions for exponential observation error decay with a constant known time delay are given. In [20] a new adaptive observer is proposed for bounded-state nonlinear systems written in triangular form. It has been shown that the observer vector gain has been obtained by updating only one parameter of the appropriate Riccati equation. However, this design is built by the knowledge of the system constant delay. Based on theory for time-delay linear systems, in [25], it has been shown how to design an observer for a class of time-delay nonlinear systems with constant time delays. Finally, in [40] different notions of parameter identifiability for nonlinear systems with known time delay has been presented.

Nonetheless, all the nonlinear observation techniques described above assume that the time delay is known ( [1], [10], [18], [20], [25], [40]).

In the present work, under unknown and variable time delay, the first main contribution is to guarantee the practical stability of the proposed observer, where the observation error converges to a ball depending on the size of the instantaneous state dynamic variation and the known upper bound delay of the unknown time-varying function delay. If the time delay is assumed to be constant and known, 
the exponential convergence of the observation error is ensured, which is a particular contribution of the main one.

The second main contribution leads to propose an analysis study, which is a natural extension of the considered class of systems without time delay. More precisely, if it is set the time delay equal to zero, all demonstrations of this paper are similar to the well-known proofs of [14] and [16]. The proposed Lyapunov-Krasovskii functional (see [22] for more details), when the time delay is taken into account, contains a term which allows to cancel the influence of the time delay on the observation error.

The paper is organized as follows. The system description is presented in Sections II. In Section III, the observer design and its convergence analysis for a class of time-delay nonlinear systems in triangular form are given. Following in Section IV, simulation results highlight the performances of the proposed observer. Finally, in Section VI, some concluding remarks are given.

\section{SYSTEM DESCRIPTION}

Consider the following class of time-delay nonlinear systems in triangular form

$$
\Sigma_{\tau(t)}:\left\{\begin{array}{l}
\dot{x}(t)=A x(t)+\Psi\left(x(t), x_{\tau(t)}, u(t), u_{\tau(t)}\right), \quad t \geq 0 . \\
y(t)=C x(t), \\
x(s)=\varphi(s), \quad \forall s \in\left[-\tau^{*}, 0\right]
\end{array}\right.
$$

where $x(t) \in R^{n}$ is the state of the system, $u(t) \in R^{m}$ is the input, $y(t) \in R$ represents the output of the system. $x_{\tau(t)}=x(t-\tau(t))$ and $u_{\tau(t)}=u(t-\tau(t))$ are respectively the delayed state and input and

$$
x=\left(\begin{array}{c}
x_{1} \\
\vdots \\
x_{n}
\end{array}\right), x_{\tau(t)}=\left(\begin{array}{c}
x_{1, \tau(t)} \\
\vdots \\
x_{n, \tau(t)}
\end{array}\right), A=\left(\begin{array}{cc}
0 & I_{n-1} \\
0 & 0
\end{array}\right), C=\left(\begin{array}{ccc}
1 & \cdots & 0
\end{array}\right)
$$

where $x_{i, \tau(t)}=x_{i}(t-\tau(t))$, for $i=1, \ldots, n$ and $I_{n-1}$ is the identity matrix of dimension n-1.

Furthermore, $\tau(t)$ represents a positive real-value unknown function that denotes the time varying 
delay affecting both state and input of the system. $\tau^{*}$ denotes the known upper bound of $\tau(t)$, and $x(s)=\varphi(s), \forall s \in\left[-\tau^{*}, 0\right]$ is an unknown continuous bounded initial function.

The vector function $\Psi\left(x, x_{\tau(t)}, u, u_{\tau(t)}\right)$ is given by

$$
\Psi\left(x, x_{\tau(t)}, u, u_{\tau(t)}\right)=\left(\begin{array}{c}
\Psi_{1}\left(x_{1}, x_{1, \tau(t)}, u, u_{\tau(t)}\right) \\
\Psi_{2}\left(x_{1}, x_{1, \tau(t)}, x_{2}, x_{2, \tau(t)}, u, u_{\tau(t)}\right) \\
\vdots \\
\Psi_{n}\left(x, x_{\tau(t)}, u, u_{\tau(t)}\right)
\end{array}\right)
$$

where the nonlinearities $\Psi_{i}\left(x_{1}, x_{1, \tau(t)}, \ldots, x_{i}, x_{i, \tau(t)}, u, u_{\tau(t)}\right)$ have a triangular structure with respect to $x_{1}, \ldots, x_{i}$ and $x_{1, \tau(t)}, \ldots, x_{i, \tau(t)}$, for $i=1, \ldots, n$.

Considering the structure of $\Psi$ and the fact that $(A, C)$ is on observable canonical form, then the system $\Sigma_{\tau(t)}(1)$ is uniformly observable for any input and time-delayed input.

To complete the description of system $\Sigma_{\tau(t)}(1)$, the following assumptions are considered.

Case 1: Variable and unknown time delay.

A1. The state and the input are considered bounded ${ }^{1}$, that is $x(t) \in \chi \subset R^{n}$ (that is a compact subset of $R^{n}$ ) and $u(t) \in U \subset R^{m}$ (that is a subset of $R^{m}$ ).

A2. The function $\Psi\left(x, x_{\tau(t)}, u, u_{\tau(t)}\right)$ is globally Lipschitz (on $\chi$ ) w.r.t $x, x_{\tau(t)}$ and $u_{\tau(t)}$, uniformly w.r.t. $u$.

A3. The time-varying delay satisfies the following properties:

i) $\exists \tau^{*}>0$, such that $\sup (\tau(t))_{t \geq 0} \leq \tau^{*}$.

ii) $\exists \beta>0$, such that $1-\dot{\tau}(t) \geq \beta$.

Case 2: Constant and known time delay $\left(\tau(t)=\tau_{c}\right)$.

H1. The function $\Psi\left(x, x_{\tau_{c}}, u, u_{\tau_{c}}\right)$ is globally Lipschitz (on $\chi \subseteq R^{n}$ : compact) w.r.t $x$ and $x_{\tau_{c}}$, and uniformly w.r.t. $u$ and $u_{\tau_{c}}$.

The class of systems $\Sigma_{\tau(t)}(1)$ under consideration includes several systems of many application do-

${ }^{1}$ The boundedness of the state excludes implicitly all initial conditions that generate unbounded state. 
mains. For instance:

- Biological: Predator-Prey Interaction (see for example [15], page 68)

- Traffic: Autonomous Intelligent Vehicle Control (see for example [29], page 55)

- Telemanipulation: Shared Compliance Control (see for example [29], page 60).

\section{OBSERVER DESIGN}

Consider system (1), then an observer for the class of systems of the form (1) is given by

$$
O_{\tau^{*}}:\left\{\begin{array}{l}
\dot{z}(t)=A z(t)+\Psi\left(z(t), z_{\tau^{*}}, u(t), u_{\tau^{*}}\right)-\theta \Delta_{\theta}^{-1} S^{-1} C^{T}\{\hat{y}(t)-y(t)\} \\
\widehat{y}(t)=C z(t)
\end{array}\right.
$$

where $\Delta_{\theta}=\operatorname{diag}\left(1, \frac{1}{\theta}, \ldots, \frac{1}{\theta^{n-1}}\right)$ with $\theta>0$ is a design parameter and $S$ is the unique symmetric positive definite solution (see for example [10], [16], [17]) of equation (3)

$$
S+A^{T} S+S A-C^{T} C=0 .
$$

Remark 1: For seek of simplicity, hereinafter we have chosen the arbitrarily fixed time-delay observer (2) equal to $\tau^{*}$. Nevertheless, for example in network applications, sometimes the delay is unknown but its upper bound and its distribution are known. In this case, the expected value of the distribution $\tau_{e}$ can be chosen as the arbitrarily fixed delay of the observer (see [36]).

Let us now define $\varepsilon=z-x$ the observation error, whose dynamics is

$$
\dot{\varepsilon}=\left\{A-\theta \Delta_{\theta}^{-1} S^{-1} C^{T} C\right\} \varepsilon+\Psi\left(z, z_{\tau^{*}}, u, u_{\tau^{*}}\right)-\Psi\left(x, x_{\tau(t)}, u, u_{\tau(t)}\right)
$$

Theorem 1: Suppose that assumptions A1-A3 are fulfilled and $\|\varepsilon(s)\|<\delta_{1}$ for any bounded $\delta_{1}>0$ and $\forall s \in\left[-\tau^{*}, 0\right]$. Then, $\exists \theta_{0} \geq 1$ such that the observation error dynamics (4) is $\delta_{2}$-practically stable $^{2}$ for all $\theta \geq \theta_{0}$ and for some bounded $\delta_{2}>0$.

\footnotetext{
${ }^{2}$ Roughly speaking, practical stability means that the observation error converges exponentially to a ball $B_{r}$ with radius $r>0$.
} For more details, see Appendix A. 
Proof: First, consider the following change of variable $e=\Delta_{\theta} \varepsilon$ and identities $\Delta_{\theta} A \Delta_{\theta}^{-1}=\theta A$, $C=C \Delta_{\theta}^{-1}$, then system (4) can be expressed in terms of $e$ as follows

$$
\dot{e}=\theta\left\{A-S^{-1} C^{T} C\right\} e+\Delta_{\theta}\left\{\Psi\left(z, z_{\tau^{*}}, u, u_{\tau^{*}}\right)-\Psi\left(x, x_{\tau(t)}, u, u_{\tau(t)}\right)\right\}
$$

In order to invoke assumptions $\mathrm{A} 1$ and $\mathrm{A} 2$, the term $\left\{\Psi\left(z, z_{\tau^{*}}, u, u_{\tau^{*}}\right)-\Psi\left(x, x_{\tau(t)}, u, u_{\tau(t)}\right)\right\}$ is rewritten as follows by adding and substracting $\Psi\left(x, x_{\tau^{*}}, u, u_{\tau^{*}}\right)$

$\Psi\left(z, z_{\tau^{*}}, u, u_{\tau^{*}}\right)-\Psi\left(x, x_{\tau(t)}, u, u_{\tau(t)}\right)=\Psi\left(z, z_{\tau^{*}}, u, u_{\tau^{*}}\right)-\Psi\left(x, x_{\tau^{*}}, u, u_{\tau^{*}}\right)+\bar{\Psi}\left(x, x_{\tau^{*}}, u, u_{\tau^{*}}, x_{\tau(t)}, u_{\tau(t)}\right)$

where

$$
\bar{\Psi}\left(x, x_{\tau^{*}}, u, u_{\tau^{*}}, x_{\tau(t)}, u_{\tau(t)}\right):=\Psi\left(x, x_{\tau^{*}}, u, u_{\tau^{*}}\right)-\Psi\left(x, x_{\tau(t)}, u, u_{\tau(t)}\right)
$$

characterizes the difference between the term that depends on the upper bound of the unknown delay and the term which depends on the unknown delay.

Define the Lyapunov-Krasovskii candidate functional

$$
V(e)=e^{T} S e+\int_{t-\tau(t)}^{t} \exp ^{-\frac{\alpha}{2 \tau^{*}}(t-\sigma)} e^{T}(\sigma) e(\sigma) d \sigma
$$

with $\alpha$ a positive constant defined thereafter. Taking the time derivative of (7) along the trajectories of system (5) and making use of (3), we have

$$
\begin{aligned}
\dot{V}(e)+\frac{\alpha}{2 \tau^{*}} V(e) & \leq-\left(\theta-\frac{\alpha}{2 \tau^{*}}\right) e^{T} S e-\theta e^{T} C^{T} C e+e^{T} e-(1-\dot{\tau}(t)) e_{\tau(t)}^{T} e_{\tau(t)} e x p^{-\frac{\alpha \tau(t)}{2 \tau^{*}}} \\
& +2 e^{T} S \Delta_{\theta}\left\{\Psi\left(z, z_{\tau^{*}}, u, u_{\tau^{*}}\right)-\Psi\left(x, x_{\tau^{*}}, u, u_{\tau^{*}}\right)\right\} \\
& +2 e^{T} S \Delta_{\theta} \bar{\Psi}\left(x, x_{\tau^{*}}, u, u_{\tau^{*}}, x_{\tau(t)}, u_{\tau(t)}\right)
\end{aligned}
$$

The following inequalities hold globally (on $\chi$ ) thanks to assumption A2

$$
\begin{gathered}
\left\|\Delta_{\theta}\left\{\Psi\left(z, z_{\tau^{*}}, u, u_{\tau^{*}}\right)-\Psi\left(x, x_{\tau^{*}}, u, u_{\tau^{*}}\right)\right\}\right\| \leq \nu\left\|\Delta_{\theta}(z-x)\right\|+\nu\left\|\Delta_{\theta}\left(z_{\tau^{*}}-x_{\tau^{*}}\right)\right\| \leq \nu\|e\|+\nu\left\|e_{\tau^{*}}\right\| \\
\left\|\Delta_{\theta} \bar{\Psi}\left(x, x_{\tau^{*}}, u, u_{\tau^{*}}, x_{\tau(t)}, u_{\tau(t)}\right)\right\| \leq \nu_{0}\left\|x_{\tau^{*}}-x_{\tau(t)}\right\|+\nu_{0}\left\|u_{\tau^{*}}-u_{\tau(t)}\right\|
\end{gathered}
$$

where $\nu$ is a Lipschitz constant in (9), and $\nu_{0}>\left\|\Delta_{\theta}\right\| \nu_{\bar{\Psi}}$, with $\nu_{\bar{\Psi}}$ is a Lipschitz constant of $\bar{\Psi}$, in (10). From assumption A1, there exists a bounded constant $\nu_{1}>\nu_{0} \nu_{x u}$ such that (10) can be written as

$$
\left\|\Delta_{\theta} \bar{\Psi}\left(x, x_{\tau^{*}}, u, u_{\tau^{*}}, x_{\tau(t)}, u_{\tau(t)}\right)\right\| \leq \nu_{1}
$$


where $\nu_{x u}$ is a positive constant which refers to the boundedness of $\left\|x_{\tau^{*}}-x_{\tau(t)}\right\|+\left\|u_{\tau^{*}}-u_{\tau(t)}\right\|$. Next, writing (8) in terms of $\|e\|$ and $\left\|e_{\tau^{*}}\right\|$, we have the following inequality

$$
\lambda_{1} e^{T}(t) e(t) \leq e^{T}(t) S e(t) \leq \lambda_{2} e^{T}(t) e(t)
$$

where $\lambda_{\min }(S):=\lambda_{1}>0$ and $\lambda_{\max }(S):=\lambda_{2}>0$ are respectively, the minimum and maximum eigenvalues $^{3}$ of $S$ and $\|S\|_{2}$ is the 2-norm matrix of $S$ satisfying $\|S\|_{2}=\vartheta>0$.

Taking into account (9), (11), (12) and using assumptions A3 i) and A3 ii), then (8) can be expressed as

$$
\dot{V}(e)+\frac{\alpha}{2 \tau^{*}} V(e) \leq-\rho_{1}(\theta, \alpha)\|e\|^{2}+\rho_{2}\|e\|\left\|e_{\tau^{*}}\right\|-\beta\left\|e_{\tau^{*}}\right\|^{2} \exp ^{-\frac{\alpha}{2}}+\mu_{1}\|e\|
$$

where $\rho_{1}(\theta, \alpha)=\lambda_{1}\left(\theta-\frac{\alpha}{2 \tau^{*}}\right)-\left\{1+2 \lambda_{2} \nu\right\}, \rho_{2}=2 \lambda_{2} \nu, \mu_{1}=2 \nu_{1} \vartheta$.

Furthermore, using the following inequality $\mu_{1}\|e\|<\frac{\eta}{2}\|e\|^{2}+\frac{1}{2 \eta} \mu_{1}^{2}<0$ with $\eta \in(0,1)$, (13) can be expressed only in function of quadratic errors terms. It follows that

$$
\dot{V}(e)+\frac{\alpha}{2 \tau^{*}} V(e)-\frac{1}{2 \eta} \mu_{1}^{2} \leq-\left(\rho_{1}(\theta, \alpha)-\frac{\eta}{2}\right)\|e\|^{2}+\rho_{2}\|e\|\left\|e_{\tau^{*}}\right\|-\beta\left\|e_{\tau^{*}}\right\|^{2} e x p^{-\frac{\alpha}{2}}
$$

Now, the right side of the above inequality can be rewritten as follows

$$
\begin{array}{r}
-\left(\rho_{1}(\theta, \alpha)-\frac{\eta}{2}-\frac{\rho_{2}^{2}}{4 \beta \exp ^{-\frac{\alpha}{2}}}\right)\|e\|^{2}-\frac{\rho_{2}^{2}}{4 \beta \exp ^{-\frac{\alpha}{2}}}\|e\|^{2}+\rho_{2}\|e\|\left\|e_{\tau^{*}}\right\|-\beta\left\|e_{\tau^{*}}\right\|^{2} \exp ^{-\frac{\alpha}{2}} \\
=-\left(\rho_{1}(\theta, \alpha)-\frac{\eta}{2}-\frac{\rho_{2}^{2}}{4 \beta} \exp ^{\frac{\alpha}{2}}\right)\|e\|^{2}-\left\{\frac{\rho_{2}}{2 \sqrt{\beta} \exp ^{-\frac{\alpha}{4}}}\|e\|-\sqrt{\beta}\left\|e_{\tau^{*}}\right\| e x p^{-\frac{\alpha}{4}}\right\}^{2}
\end{array}
$$

To satisfy inequality (14), all we need to do is choose $\alpha$ and $\theta$ such that $\left(\rho_{1}(\theta, \alpha)-\frac{\eta}{2}-\frac{\rho_{2}^{2}}{4 \beta} \exp ^{\frac{\alpha}{2}}\right)>0$. Set $\alpha=\frac{2}{q} \ln \theta$. Then, $\exists \theta_{0} \geq 1$ such that the following inequality is verified

$$
\theta-\frac{1}{q \tau^{*}} \ln \theta-\frac{\nu^{2} \lambda_{2}^{2}}{\lambda_{1} \beta} \sqrt[q]{\theta}-\frac{\eta}{2 \lambda_{1}}-\frac{1+2 \lambda_{2} \nu}{\lambda_{1}}>0
$$

where $\theta>\theta_{0}$ and $q \geq 2$. Thereafter, (14) becomes $\dot{V}(e) \leq-\frac{\ln \theta}{q \tau^{*}} V(e)+\frac{\mu_{1}^{2}}{2 \eta}$, which is equivalent to

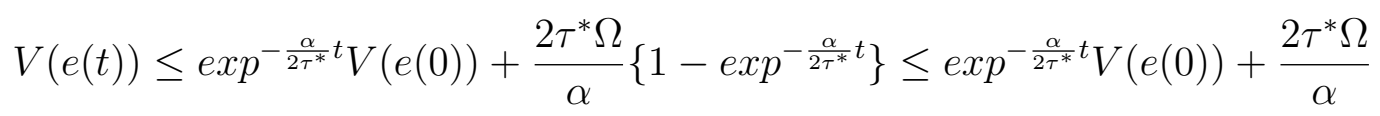

\footnotetext{
${ }^{3} \lambda_{1}$ and $\lambda_{2}$ are obtained directly by solving equation (3), as A and C are given explicitly depending on the dimension $n$ of the system.
} 
where $\Omega=\frac{\mu_{1}^{2}}{2 \eta}$.

Now, the objective is to prove the uniform practical stability of (4). For that, (16) should be rewritten in terms of the observation error norm. Then, from Lemma 1 (see Appendix B), the following inequality is obtained

$$
\begin{aligned}
V(e(t)) & \leq \lambda_{2}\|e(t)\|^{2}+\delta_{M}\left(\alpha, \tau^{*}\right) \max _{s \in\left[t-\tau^{*}, t\right]}\|e(s)\|^{2} \\
& \leq \delta_{M}^{*}\left(\alpha, \tau^{*}\right) \max _{s \in\left[t-\tau^{*}, t\right]}\|e(s)\|^{2} .
\end{aligned}
$$

where $\delta_{M}^{*}\left(\alpha, \tau^{*}\right)=\lambda_{2}+\delta_{M}\left(\alpha, \tau^{*}\right)$ with $\delta_{M}\left(\alpha, \tau^{*}\right)=\frac{2 \tau^{*}\left(1-\exp p^{-\frac{\alpha}{2}}\right)}{\alpha}$.

By using (12) and (17), it follows that

$$
\begin{aligned}
\lambda_{1}\|e(t)\|^{2} \leq V(e(t)) & \leq \exp ^{-\frac{\alpha}{2 \tau^{*} t} V(e(0))+\frac{2 \tau^{*} \Omega}{\alpha}} \\
& \leq \delta_{M}^{*}\left(\alpha, \tau^{*}\right) \exp ^{-\frac{\alpha}{2 \tau^{*}} t} \max _{s \in\left[-\tau^{*}, 0\right]}\|e(s)\|^{2}+\frac{2 \tau^{*} \Omega}{\alpha}
\end{aligned}
$$

where an upper bound $V(e(0))$ comes from (17) by setting $t=0$.

Consequently, (18) can be written in terms of $\|e(t)\|$, as follows

$$
\|e(t)\| \leq K\left(\alpha, \tau^{*}\right) \exp ^{-\frac{\alpha}{4 \tau^{*}} t} \max _{s \in\left[-\tau_{c}, 0\right]}\|e(s)\|+\Gamma
$$

with $K\left(\alpha, \tau^{*}\right)=\sqrt{\frac{\delta_{M}^{*}\left(\alpha, \tau^{*}\right)}{\lambda_{1}}}$ and $\Gamma=\sqrt{\frac{2 \tau^{*} \Omega}{\alpha \lambda_{1}}}$.

Then, there exist $\delta_{1}>0, \delta_{2}>0$ and $T_{0}>0$, such that

$$
\|e(t)\| \leq K\left(\alpha, \tau^{*}\right) \exp ^{-\frac{\alpha}{4 \tau^{*}} T_{0}} \delta_{1}+\Gamma \leq \delta_{2}, \text { for } \quad \forall t \geq T_{0}
$$

where $\max _{s \in\left[-\tau^{*}, 0\right]}\|e(s)\| \leq \delta_{1}$. Finally, from the change of variable $e=\Delta_{\theta} \varepsilon$, the observation error $\varepsilon(t)$ satisfies

$$
\|\varepsilon(t)\| \leq \theta^{n-1}\|e(t)\| \leq \theta^{n-1} \delta_{2}
$$

where $\delta_{2}$ corresponds to parameter $\zeta$ in the $\zeta$-practical stability (see Appendix A for more details).

Then, we can conclude that observation error (20) is globally (on $\chi$ ) $\delta_{2}$-practically stable. This ends the proof of Theorem 1 . 
Corollary 1: Let $\tau_{c}$ be a known constant time delay. Consider system (1) with $\tau(t)=\tau_{c}$ and Hypothesis H1 holds. Then, system (2) with $\tau^{*}=\tau_{c}$ is a globally (on $\chi$ ) exponential observer ${ }^{4}$ for system (1).

Proof: $\quad$ Let $\varepsilon=z-x$ and $\tau(t)=\tau^{*}=\tau_{c}$. Following the proof of Theorem 1, inequality (8) becomes

$$
\begin{aligned}
\dot{V}(e)+\frac{\alpha}{2 \tau_{c}} V(e) & \leq-\left(\theta-\frac{\alpha}{2 \tau_{c}}\right) e^{T} S e-\theta e^{T} C^{T} C e+e^{T} e-e_{\tau_{c}}^{T} e_{\tau_{c}} e x p^{-\frac{\alpha}{2}} \\
& +2 e^{T} S \Delta_{\theta}\left\{\Psi\left(z, z_{\tau_{c}}, u, u_{\tau_{c}}\right)-\Psi\left(x, x_{\tau_{c}}, u, u_{\tau_{c}}\right)\right\}
\end{aligned}
$$

where the term (6) is equal to zero $\left(\tau(t)=\tau^{*}\right)$, which implies that $\mu_{1}=0$ in (13) and consequently, $\Omega=0$ in $(16)$.

Then, from Hypothesis H1, the following inequality holds globally (on $\chi$ )

$$
\left\|\Delta_{\theta}\left\{\Psi\left(z, z_{\tau_{c}}, u, u_{\tau_{c}}\right)-\Psi\left(x, x_{\tau_{c}}, u, u_{\tau_{c}}\right)\right\}\right\| \leq \kappa\left\|\Delta_{\theta}(z-x)\right\|+\kappa\left\|\Delta_{\theta}\left(z_{\tau_{c}}-x_{\tau_{c}}\right)\right\| \leq \kappa\|e\|+\kappa\left\|e_{\tau_{c}}\right\|
$$

where $\kappa$ is a Lipschitz constant.

By using (22) in (21) and after some computations, we get $\dot{V}(e)+\frac{\alpha}{2 \tau_{c}} V(e) \leq 0$, which is equivalent to $V(e(t)) \leq \exp ^{-\frac{\alpha}{2 \tau_{c}} t} V(e(0))$ where $\alpha=\frac{2}{q} \ln \theta$. The design parameter $\theta$ is obtained from (15), without $-\frac{\eta}{2 \lambda_{1}}$ and by setting $\beta=1$ and $\nu=\kappa$. Following the same procedure proof of Theorem 1 and using Lemma 1 on Appendix $\mathrm{B}$, the observation error $e(t)$ is

$$
\|e(t)\| \leq \sqrt{\frac{\delta_{M}^{*}\left(\alpha, \tau_{c}\right)}{\lambda_{1}}} \exp ^{-\frac{\alpha}{4 \tau_{c}} t} \max _{s \in\left[-\tau_{c}, 0\right]}\|e(s)\|
$$

where $\delta_{M}^{*}\left(\alpha, \tau_{c}\right)=\lambda_{2}+\delta_{M}\left(\alpha, \tau_{c}\right)$. Finally, with $e=\Delta_{\theta} \varepsilon$, the observation error $\varepsilon(t)$ is given by

$$
\|\varepsilon(t)\| \leq \theta^{n-1} K\left(\alpha, \tau_{c}\right) \exp ^{-\frac{\alpha}{4 \tau_{c}} t} \max _{s \in\left[-\tau_{c}, 0\right]}\|\varepsilon(s)\|
$$

which is, from Hypothesis H1, globally (on $\chi$ ) exponential stable, where $K\left(\alpha, \tau_{c}\right)=\sqrt{\frac{\delta_{M}^{*}\left(\alpha, \tau_{c}\right)}{\lambda_{1}}}$. This ends the proof of Corollary 1.

${ }^{4}$ Exponential observer means that the observation error converges exponentially to zero. 


\section{Illustrating EXAMPLE}

Let us consider the unknown time-variable delay nonlinear system

$$
\Sigma_{\tau(t)}:\left\{\begin{aligned}
\dot{x}_{1} & =-\gamma_{1} x_{1, \tau(t)}^{2}+x_{2} \\
\dot{x}_{2} & =-x_{1} u_{\tau(t)}-\gamma_{2} x_{1} x_{2, \tau(t)} \\
y & =x_{1}
\end{aligned}\right.
$$

where $A=\left(\begin{array}{cc}0 & 1 \\ 0 & 0\end{array}\right) ; \Psi\left(x, x_{\tau(t)}, u, u_{\tau(t)}\right)=\left(\begin{array}{c}-\gamma_{1} x_{1, \tau(t)}^{2} \\ -x_{1} u_{\tau(t)}-\gamma_{2} x_{1} x_{2, \tau(t)}\end{array}\right)$, with $\gamma_{1}=\gamma_{2}=0.01$. From this definition of $\Psi A 2$ assumption is satisfied. The input $u=\sin (2 \pi f t)$ with $f=50 H z$. It is bounded and from figures (1) and (2) the states are bounded, which make assumption A1 holds. The function $\tau(t)$ is defined as follows:

$$
\tau(t)=\left\{\begin{array}{rlll}
0 & \text { if } & t \in[0,5] \\
2\left(\frac{t}{5}-1\right) & \text { if } & t \in[5,10] \\
2 & \text { if } & t \in[10,20] \\
2\left(-\frac{2 t}{5}+10\right) & \text { if } & t \in[20,25] \\
0 & \text { if } & t \in[25,35]
\end{array}\right.
$$

where the assumption A3 is verified with $\tau^{*}=2 \mathrm{~s}$. For system (23), an observer $O_{\tau^{*}}$ is designed as (2), with

$$
z=\left(\begin{array}{c}
z_{1} \\
z_{2}
\end{array}\right) \text { and } \Psi\left(z, z_{\tau^{*}}, u, u_{\tau^{*}}\right)=\left(\begin{array}{c}
-\gamma_{1} z_{1, \tau^{*}}^{2} \\
-z_{1} u_{\tau^{*}}-\gamma_{2} z_{1} z_{2, \tau^{*}}
\end{array}\right)
$$

The initial conditions for the system are $x(0)=[2,1]^{T}$, for the observer $z(0)=[1,2]^{T} . \theta=10$.

If the time delay is constant and known, that is $\tau(t)=\tau^{*}=\tau_{c}$, then assumption $H 1$ is satisfied.

Fig. 1 and Fig. 2 show the obtained simulation results. According to the practical stability enhanced in the proof of Theorem 1, it can be seen that the observation error (Fig. 1 and Fig. 2) of the observer converges to a ball with a radius $r>0$ depending on two parameters: the size of the instantaneous 
state dynamic variation and the delay difference between the observer and the system. For example, at $t=70 \mathrm{~s}$ (Fig. 1 and Fig. 2) the system exhibits a highest variation of the instantaneous bounded state dynamic with the maximum bounded delay difference between the observer and the system, then a bounded highest error appears. When the delay is the same for the system and the observer $\tau(t)=\tau_{c}=\tau^{*}$, for example from $t=10 \mathrm{~s}$ to $t=20 \mathrm{~s}$ (Fig. 1 and Fig. 2), the observation error is cancelled and an exponential stability is ensured according to the proof of Corollary 1.


Fig. 1. $x_{2}$ and its estimate $z_{2}$, time-varying delay $\tau(t)$ and observation error $\left(z_{2}-x_{2}\right)$. 



Fig. 2. $x_{1}$ and its estimate $z_{1}$, time-varying delay $\tau(t)$ and observation error $\left(z_{1}-x_{1}\right)$.

\section{Conclusion}

In this paper, an observer for a class of time-delay nonlinear systems in strictly lower triangular form was proposed. In the general case of a bounded and unknown variable time delay, sufficient conditions were given to guarantee a practical stability of the proposed observer. Furthermore, the exponential convergence of the observer was proved, being a particular case of the general one when a constant known time delay is considered. Simulation results were presented for both cases in order to illustrate the well performances of the proposed observer. 


\section{APPENDIX}

\section{A. Practical Stability}

The definition of practical stability for a general class of time-delay systems is introduced. The practical stability definition is more suitable in dealing with problems in the real world. Then, for practical purpose, practical stability seems desirable (see [23] for systems without delays and [38] for time-delay systems). It is worth mentioning that the practical stability is refereed to as ultimate boundedness with a fixed bound. Now, we establish the definition for a general class of time-delay systems of the form

$$
\left\{\begin{array}{l}
\dot{x}=f(t, x, x(t-\tau)), \quad t>0 \\
x(s)=\Phi(s), \quad \forall s \in[-\tau, 0] .
\end{array}\right.
$$

where $x(t, \Phi)$ is the solution of the system with initial function $\Phi$, verifying

$$
x(s, \Phi)=\Phi(s), \quad \forall s \in[-\tau, 0] .
$$

$\Phi$ is a continous function in the Banach space $C_{n, \tau}:=C\left([-\tau, 0], R_{n}\right)$ with norm

$$
\|\Phi\|_{\tau}:=\max _{s \in[-\tau, 0]}\|\Phi(s)\|
$$

$f: R_{+} \times R^{n} \times R^{n} \rightarrow R^{n}$ is piecewise continuous in $t$ and locally Lipschitz in $x$ and $\tau$ is the delay.

Definition 1: (Practical stability of time-delay systems)

The system (25) is said to be $\zeta$-practically stable, if for some $\zeta>0$, there exists $T_{0}=T_{0}(\zeta, \Phi)$, such that

$$
\|x(t)\| \leq K \exp ^{-\beta T_{0}}\|\Phi\|_{\tau}+r \leq \zeta, \quad \forall t \geq T_{0}
$$

with $r>0, K>0$ and $\beta>0$.

Remark 2: The practical stability is refereed as uniformly exponentially convergent to a ball $B_{r}$ with radius $r>0$. 


\section{B. Technical result}

Lemma 1: Consider function

$$
\int_{t-\tau^{*}}^{t} \exp ^{-\frac{\alpha}{2 \tau^{*}}(t-\sigma)}\|e(\sigma)\|^{2} d \sigma>0
$$

Then, it satisfies the inequality

$$
\int_{t-\tau^{*}}^{t} \exp ^{-\frac{\alpha}{2 \tau^{*}}(t-\sigma)}\|e(\sigma)\|^{2} d \sigma<\delta_{M}\left(\alpha, \tau^{*}\right) \max _{s \in\left[t-\tau^{*}, t\right]}\|e(s)\|^{2}
$$

Proof: Consider (7) and using Hölder inequality, we obtain

$$
\int_{t-\tau^{*}}^{t} \exp ^{-\frac{\alpha}{2 \tau^{*}}(t-\sigma)}\|e(\sigma)\|^{2} d \sigma \leq \max _{s \in\left[t-\tau^{*}, t\right]}\|e(s)\|^{2} \int_{t-\tau^{*}}^{t} \exp ^{-\frac{\alpha}{2 \tau^{*}}(t-\sigma)} d \sigma .
$$

Furthermore, with $\delta_{M}\left(\alpha, \tau^{*}\right)$ defined in (17), inequality (27) follows.

\section{REFERENCES}

[1] M. Anguelova and B. Wennberga, "State elimination and identifiability of the delay parameter for nonlinear time delay systems", Automatica, Vol. 44, N 5, pp. 1373-1378, 2008.

[2] J. Anthonis, Seuret A., J-P. Richard and H. Ramon, "Design of a pressure control system with band time delay", IEEE Transactions on Control Systems Technology, Vol. 15, No 3, 2007.

[3] L. Belkoura, J-P. Richard and M. Fliess, "Parameters estimation of systems with delayed and structured entries", Automatica, Vol. 45, $\mathrm{N}^{\circ}$ 5, pp. 1117-1125, 2009.

[4] K. Bhat and H. Koivo, "An observer theory for time delay systems", IEEE TAC, Vol. 21, No 2, pp. 266-269, 1976.

[5] M. Boutayeb, "Observers design for linear time-delay systems", Systems \& Control Letters, Vol. 44, No 2, pp. 103-109, 2001.

[6] H. Ch. Cho and J. H. Park, "Stable bilateral teleoperation under a time delay using a robust impedance control", Mechatronics, Vol. 15, No 5, pp. 611-625, 2005.

[7] H. H. Choi and M. J. Chung, "Observer-based H controller design for state delayed linear systems", Automatica, Vol. 32, $\mathrm{N}^{\circ}$ 7, pp. 1073-1075, 1996.

[8] M. Darouach, "Linear functional observers for systems with delays in the state variables". IEEE TAC, Vol. 46, N ${ }^{o} 3$, pp. 491-497, March 2001.

[9] K.K. Fan and J.G. Hsieh, "LMI Approach to design of robust state observer for uncertain systems with time-delay perturbation". In IEEE ICIT, 2002.

[10] M. Farza, A. Sboui, E. Cherrier and M. M'Saad, "High-gain observer for a class of time-delay nonlinear systems", Int. J. Control, Vol. 83, $\mathrm{N}^{o}$ 2, pp. 273-280; 2010.

[11] M. Fliess and H. Mounier, "Controllability and observability of linear delay systems: an algebraic approach", ESAIM Control Optim. Calc. Var., Vol. 3, pp. 301-314, 1998. 
[12] M. Fliess, C. Join and M. Mboup, "Algebraic change-point detection", Applicable Algebra in Engineering, Communication, and Computing, Vol. 21, $\mathrm{N}^{\circ}$ 2, pp. 131-143, 2010.

[13] E. Fridman, F. Gouaisbaut, M. Dambrine and J-P. Richard, "A descriptor approach to sliding mode control of systems with time-varying delays", International Journal of Systems Science, Vol. 34, Nº 8-9, pp. 553-559, 2003.

[14] E. Fridman, "Stability of systems with uncertain delays: a new 'complete' Lyapunov-Krasovskii functional", IEEE TAC, Vol. $51, \mathrm{~N}^{o} 5$, pp. 885-890, 2006

[15] J-E. Fonde, "Delay differential equation models in mathematical biology", PHD, University of Michigan, 2005.

[16] J.P. Gauthier, H. Hammouri and S. Othman, "A simple observer for nonlinear systems. Applications to bioreactors", IEEE TAC, Vol. 37, Nº 6, 875-880, 1992.

[17] M. Ghanes, J.B. Barbot, J. DeLeon, A. Glumineau, "A robust output feedback controller of the induction motor drives: new design and experimental validation", International Journal of Control, Vol. 83, Nº 3, pp. 484-497, 2010.

[18] A. Germani, C. Manes and P. Pepe, "An Asymptotic State Observer for a Class of Nonlinear Delay Systems", Kybernetika, Vol. 37, $\mathrm{N}^{\circ} 4$, pp. 459-478, 2001.

[19] M. Hou and R. T. Patton, "An observer design for linear time-delay systems", IEEE TAC, Vol. 47, No 1, pp. 121-125, 2002.

[20] S. Ibrir. "Adaptive observers for time delay nonlinear systems in triangular form", Automatica, Vol. 45, N 10, pp. 2392-2399, 2009

[21] V. L. Kharitonov and D. Hinrichsen, "Exponential estimates for time delay systems", Syst. Control Lett., Vol. 53, N ${ }^{o}$ 5, pp. $395-405,2004$

[22] N.N. Krasovskii, "On the analytical construction of an optimal control in a system with time lags", J. Appl. Math. Mech., Vol. 26, $\mathrm{N}^{o} 1$, pp. 50-67, 1962.

[23] V. Laskhmikanthan, S. Leela and A. Martynyuk, "Practical stability of nonlinear systems". In Word Scientific, 1990.

[24] N. MacDonald, "Time Lags in Biological Models". In Lecture Notes in Biomath. Springer, 1978.

[25] L.A. Marquez-Martinez, C.H. Moog, and V.V. Martin, "Observability and observers for nonlinear systems with time delays", Kybernetika, Vol. 38, $N^{o}$ 4, pp. 445-456, 2002.

[26] H. Mounier and J. Rudolph, "Flatness Based Control of Nonlinear Delay Systems: A Chemical Reactor Example", Int. J. Control, Vol. 71, $N^{o}$ 5, pp. 871-890, 1998.

[27] K. Natori and K. Ohnishi, "A design method of communication disturbance observer for time-delay compensation, taking the dynamic property of network disturbance into account", IEEE T. Indus. Electronics, Vol. 55, $N^{\circ}$ 5, pp. 2152-2168, 2008.

[28] S-I. Niculescu, C-E. de Souza, L. Dugard and J-M. Dion, "Robust exponential Stability of uncertain systems with time-varying delays", IEEE TAC, Vol. 43, $N^{o}$ 5, pp. 743-748, 1998.

[29] S-I. Niculescu, "Delay effects on stability: A robust control approach", Springer LNCIS, 2001.

[30] P. Picard, O. Sename and J.F. Lafay. "Observers and observability indices for linear systems with delays". In: CESA 96, IEEE Conference on Computational Engineering in Systems Applications, volume 1, pp. 81-86, 1996.

[31] J-P. Richard, "Time-delay systems: an overview of some recent advances and open problems", Automatica, Vol. 39, $N^{o} 10$, pp. 1667-1694, 2003. 
[32] J-P. Richard, F. Gouaisbaut and W. Perruquetti, "Sliding mode control in the presence of delay. Kybernetica, Vol. $37, N^{o} 4$ , pp. 277-294, 2001.

[33] O. Sename, "New trends in design of observers for time-delay systems". Kybernetica, Vol. 37, $N^{o}$ 4, pp. 427-458, 2001.

[34] O. Sename and C. Briat. "H1 observer design for uncertain time-delay systems". In IEEE ECC, 2007.

[35] A. Seuret, T. Floquet, J.-P. Richard, and S.K. Spurgeon, "A sliding mode observer for linear systems with unknown timevarying delay". In IEEE ACC, 2007.

[36] A. Seuret, T. Floquet, J.-P. Richard, and S.K. Spurgeon, "Topics in Time-Delay Systems: Analysis, Algorithms and Control". In Springer Verlag (Ed.), 2008.

[37] E. Shustin, L. Fridman, E. Fridman and F. Castaos, "Robust semiglobal stabilization of the second order system by relay feedback with an uncertain variable time delay", SIAM Journal on Control and Optimization Vol. 47, $N^{\circ}$ 1, pp 196-217, 2008.

[38] R. Villafuerte, S. Mondie, Z. Poznyak, "Practical stability of time delay systems: LMI's approach". In IEEE CDC, 2008.

[39] Z. Wang, B. Huang and H. Unbehausen, "Robust $H_{\infty}$ observer design for uncertain time-delay systems:(I) the continuous case". In IFAC 14-th world congress, Beijing, China, pp. 231-236, 1999.

[40] J. Zhang, X. Xia, and C.H. Moog, "Parameter identifiability of nonlinear systems with time-delay", IEEE TAC, Vol. 47, $N^{o}$ 2, pp. 371-375, 2006.

[41] G. Zheng, J P Barbot, D. Boutat, T. Floquet and J P Richard, "On obserability of nonlinear time-delay systems with unknown inputs", IEEE Transactions on Automatic Control, Vol. 56, Nº, p.1973-1978, 2011. 